This material is presented to ensure timely dissemination of scholarly and technical work. Copyright and all rights therein are retained by authors or by other copyright holders. All persons copying this information are expected to adhere to the terms and constraints invoked by each author's copyright. In most cases, these works may not be reposted without the explicit permission of the copyright holder.

This version of the referenced work is the PRE-PRINT version of the article-it is NOT the final published version nor the corrected proofs. If you would like to receive the final published version please send a request to any of the authors and we will be happy to send you the latest version. Moreover, you can contact the publisher's website and order the final version there, as well.

The current reference for this work is as follows:

Silic, M., \& Cyr, D. (2016). Colour Arousal Effect on Users’ Decision-Making Processes in the Warning Message Context. In F.-H. F. Nah \& C.-H. Tan (Eds.), HCI in Business, Government, and Organizations: Information Systems: Third International Conference, HCIBGO 2016, Held as Part of HCI International 2016, Toronto, Canada, July 17-22, 2016, Proceedings, Part II (pp. 99-109). Cham: Springer International Publishing.

If you have any questions, would like a copy of the final version of the article, or would like copies of other articles we've published, please email any of us directly. 


\title{
Colour arousal effect on users' decision-making processes in the warning message context
}

\author{
Mario Silic ${ }^{1}$, Dianne Cyr ${ }^{2}$ \\ ${ }^{1}$ Institute of Information Management (IWI), University of St. Gallen, Switzerland/ZSEM \\ mario.silic@unisg.ch \\ ${ }^{2}$ Beedie School of Business, Simon Fraser University, Canada \\ cyr@sfu.ca
}

\begin{abstract}
This research is aimed at understanding how colour affects users' decision-making processes in the context of warning banner messages. So far, little research has examined this complex psychological and cognitive process wherein the psychology of colour can play an important role in the relationship between the warning message and the user's decision-making process. We closed the existing research gap by understanding how different colour applications (black, blue, yellow, red, green, white) influence users' decision-making processes. We built our work on the theory of psychological reversals to colour, supported by the Cognitive-Affective model of communication. We conducted an online experiment measuring actual users' behaviour of 217 participants. We found that the colour application has different arousal effects. Overall, we advance understanding of the psychological process that precedes a decision with a focus on the importance of colour.
\end{abstract}

Keywords: psychology of colour, warning banner message, user compliance

\section{Introduction}

Decision-making process is result of trust [1] and different risk factors [2-4] which will ultimately help or hinder the entire process [5] where the colour inputs play an important role. Drivers will stop at traffic red lights as red suggests that there is danger ahead. Colour affects our cognitive systems [6, 7], human perceptions, psychological and emotional reactions and ultimately, our behavioural intentions [8, 9]. The relationship between colour and cognitive functioning has mostly been studied in the field of Psychology. For instance, red was found to be linked to performance attainment [10], which suggests a strong relationship with the emotions whereby diverse psychological reactions can be observed, such as anxiety or pleasure [8]. One such example, wherein first impressions play an important role, is the online context, in 
which the majority of studies have focused on the impact of colours on website design [11-13], seeking to understand the behaviours of internet shoppers [14, 15]. Overall, past studies have tried to understand how to better capture the user's attention through more appealing website content. In the computer digital world, a warning message on the computer represents communication designed to prevent users from hurting themselves or others [16, 17]. In general, warnings have been found to be quite efficient in preventing hazards and reducing negative outcomes [18-20]. However, and similar to the real-life situation, in the computer context, digital warnings are quite often ignored. Several different explanations have been provided to explain users' noncompliant behaviour, such as: 1) users are habituated to warning messages and consequently, do not read them [21]; and 2) users ignore warnings due to their content [22, 23]. Warning standards and guidelines, such as those put forth by the ANSI - American National Standards Institute - recommend that warnings should be composed of the terms Danger, Warning or Caution combined with a specific colour - red, orange and yellow and an alert symbol [24]. Consequently, the majority of software makers have adopted these guidelines with red being used as a predominant colour. For instance, the latest versions of the Chrome and Internet Explorer web browsers use a red background to inform the user about the potential risk that the user may experience if he/she decides to continue with his/her action.

Past research has concluded that red warnings yield higher adherence rates than black or green warnings in the alcoholic beverage containers context [25]. However, only few studies have tried to understand whether red is really the most efficient colour for computer warning messages when it comes to drawing the user's attention. A study done by Egelman and Schechter [26] investigated the way in which the choice of the background colour (red vs white) in the warning affects the user's decision to comply with the warning. Overall, the number of studies that have investigated the relationship between the colour and the user's cognitive decision-making process is still relatively low. Another challenge with past studies is that they have been poorly supported by the theory. They have mostly used findings from other fields, such as the tobacco industry, in order to implement the same results in the computer warning context. This has resulted in seemingly contradictory and often inconclusive results when it comes to the efficiency of the computer warning message.

Also, the way in which colour affects the user's decision-making process over time has not been addressed by past research. In other words, an understanding of how different colours may affect the user's conscious decisions when the user is confronted by the warning message for the first time, but also during all the consecutive event occurrences, is still lacking. For instance, if the warning message colour is black, instead of red, it could be that the user, upon the third of fourth time of seeing the warning message, would be more careful and attentive to the hazard.

Overall, with this research study we aim to close the existing research gap by studying the effects of colour on the user's decision-making process. We build our work on the theory of psychological reversals to colour [27] to understand how users behave when confronted with the computer warning message. 
In the following sections we will present the theoretical background and develop our research model. Then, we will describe the approach and context chosen to empirically test our model, and report our findings. The paper concludes with a discussion on the results, implications and limitations of our study.

\section{Theoretical framework}

\subsection{Cognitive-affective model of communication}

According to the cognitive-affective model of communication suggested by Te-eni [28], there are three factors that affect the communication process: "(1) inputs to the communication process (task, sender-receiver distance, and values and norms of communication with a particular emphasis on inter-cultural communication); (2) a cognitive affective process of communication; and (3) the communication impact on action and relationship”. The model suggests that the communication medium and the form of the message will shape the receiver's behaviour. In our context, the communication medium is the warning message itself and the message form corresponds to the colour application. During the communication process, the warning message in combination with the colour application will potentially influence the user's decisionmaking process in such a way that the user will either react positively to the communication medium content or will not have any affective reaction. Overall, the model suggests that a relationship has to be established between the user and the communication medium (i.e. warning) before the user's behaviour will lead to an action. Hence, there is a balance between relationship and action, between cognition and affect, and between message and medium [28]. Another dimension that is important for the cognitive-affective model of communication is the cultural aspect. As communication action is bounded by social norms and values, users may have different expectations of how the message should be represented.

\subsection{Theory of psychological reversals}

The theory of psychological reversals [27] suggests two levels of preferred felt arousal: one high and one low. Although switching ("reversals") from high to low is possible, either a high or low level will be preferred at a certain point in time. There is a strong association between arousal preference and colour preference, whereby users will be excited and aroused by certain colours and relaxed by others. For instance, warm colours will generally provoke "active feelings" while cool colours are "much less likely to cause extreme reactions" [29]. Similarly, Gerard [30] found that when a red light was projected on a diffusing screen, an increased arousal was observed in the subjects. Clearly, long-wavelength colours (e.g. red, orange) are more stimulating compared to short-wavelength colours (e.g. blue, violet), which are more relaxing [27]. In the online shopping context, it was found that cool tones such as blue are preferred over warm tones such as red [11]. We expect, then, to see different levels of appeal to users who are expected to behave differently when presented with warm or 
cool tones. As the cognitive-affective model of communication suggests that cognition and affect will be impacted by the communication medium (i.e. warning message), in such circumstances it is likely that the user will feel either high or low arousal. Consequently, there will be a possibility that the user may switch from a high to a low level if he/she is confronted with a different colour application. This could be even more pronounced in a different cultural aspect.

\section{Hypothesis Development}

The effects of colour on human preferences have received considerable attention from psychologists [31]. Their relevance is not just related to aesthetics. In western cultures, for instance, red means "mistake" (e.g. teachers use red to correct mistakes), stop (e.g. red traffic lights) or danger (e.g. warning message). But it is also associated with love and sexuality (e.g. Amsterdam red light district). When it comes to gender separation, pink is usually attributed to girls and blue to boys [32]. White usually means immaculacy and black corresponds to tragic situations (e.g. death). Overall, different colour applications may have different impacts on the user's decisionmaking process. However, current research suggests that red is the colour that commonly represents danger, as it denotes risk and danger on warning labels, traffic signals, and many other systems used to inform about the threat (e.g. anti-virus software). However, as can be seen from previous studies, red can provide different levels of arousal. While we expect to find red to be the most efficient colour in transmitting risk in the computer warning message context, the lack of empirical findings related to colour and user decision making suggests that the relationship between colour application and the warning message needs to be better analysed. Consequently, it could be that other colours will have stronger secondary (i.e. second and all consecutive warning message displays) arousal effects on the user's attention. For instance, humans are so habituated to seeing the colour red in everyday life (e.g. traffic signs) that in the computer context the user may pay less attention (i.e. not read the warning itself) and, consequently, may skip over and ignore the message. It could be interesting to see what would happen, for instance, if the computer warning message had a constantly changing background, with the objective of decreasing the user's habituation to seeing the colour red. On the one hand, the user could be confused by this constant colour change, but on the other hand, the user might pay more attention as he/she would not become habituated to always seeing the red background. Overall, we argue that different colour applications will have high or low psychological arousal effects on the user. Hence, we hypothesize:

Hypothesis 1: Different colour applications will have different levels of psychological arousal effects on the user's actual behaviour when confronted by the computer warning message 


\section{$4 \quad$ Research design}

\subsection{Participants, Measures and procedures}

In order to test our research hypothesis, we conducted a non-controlled online experiment involving participants from US and India. Institutional Review Board (IRB) approval was given to collect data and human-subject protocols were followed. In addition, every participant had to provide his or her consent for being part of a research study. The participants were informed that the study's objective was to get their view on the photoproduct they like the most. Hence, we used deception to increase realism of the results. We recruited participants from US and India by using Amazon Mechanical Turk.

Our study uses the experiment method to explore and measure the progression, frequency, and duration (i.e. time) of user behaviour when confronted with the warning banner message through different colour applications. Users were provided with a web link, which led them to a website, where they were asked to complete a task. The task consisted of choosing the product image they prefer. After clicking on the task 'start' button, they were presented with the warning banner message (Figure 1). In order to test the frequency (i.e. the repeated effect) we displayed the warning banner message to the same participants twice - first after the second photoproduct evaluation and second after the fourth evaluation. We limited the number of warning appearances to two in order not to raise any suspicions from the participant's side. The warning message is based on the latest Google Chrome web browser malware warning, which we modified (Figure 1) slightly (instead of using the word 'malware' we used the word 'safe' for simplicity and comprehension reasons). The background colours were randomly displayed (the random function is used in the web application to control the display), and could be red, blue, green, black, yellow or white. As red is the default colour for warning message in the majority of the web browsers (e.g. Google Chrome, Internet Explore and Firefox) we use red as the control group. Further, the choice for other colours is based on the colour-culture chart as suggested by Russo and Boor [33], but also on past studies [e.g. 11], which found these colours to have a high risk component or to transmit some strong emotions and feelings.

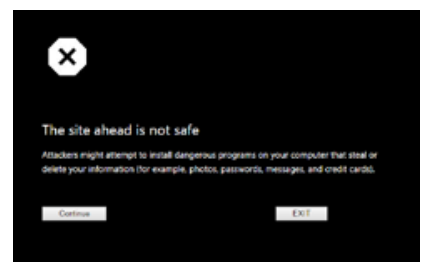

Fig. 1. Warning message displayed (adapted from Google Chrome version 46)

In order to measure users' actual behaviour we use a categorical variable (“decision”) which can take two values. When users click on 'Continue' or 'EXIT', the binary 
decision is registered on a remote SQL server ( 0 for continue and 1 for Exit) together with several other data such as: browser version, time spent deciding (time is measured in milliseconds and corresponds to the time from when the user clicked on the start button until the user clicks on continue or exit).

\subsection{Method for analysis}

To analyse the effects we used the survival techniques such as the Kaplan-Meier Survival Curve, which is an estimator used to estimate survival time from lifetime data. The Kaplan-Meier method is a nonparametric method used to estimate the probability of survival past given time points (i.e. it calculates a survival distribution) [34]. Time to event represents an event course duration, which has a beginning and an end for each user. In this type of analysis, every participant is characterized by three variables: 1) the duration, 2) the status at the end of the event (exit or continue) and 3) the colour application. Furthermore, to test the effect of the warning message on the duration (repeated use) we use Cox's proportional-hazard regression model, which allows for investigations of the relationships between the survival of the event and independent measures of interest [35].

\section{$5 \quad$ Results}

We received 435 events (both first and second occurrence) from 217 participants (one participant second warning event was not registered as participant closed web browser). Detailed demographics are presented in Table 2.

\begin{tabular}{|l|c|c|c|c|}
\hline Country & \# of users & \multicolumn{2}{|c|}{ Gender } & $\begin{array}{c}\text { Average } \\
\text { Age }\end{array}$ \\
\hline United States & 106 & 59 & 47 & 33.4 \\
\hline India & 111 & 66 & 45 & 31.8 \\
\hline
\end{tabular}

Table 1. Participant demographics

Further, in order to understand how the warning message impacts user behaviour in presence of a different colour application we analyze the survival time before the hazard occurs (i.e. user clicks on continue button in the warning banner message). As we cannot simply compare the average hazard durations, due to the right skewed distribution of the survival time, we use survival and event history analysis techniques. Event history analysis is a statistical method that deals with timing and duration until the occurrence of an event. The method examines the hazard rate that calculates conditional probability that an event occurs at a particular time interval. In other words, how long it takes the event to occur. To start with, Table 3 provides an overview of the case summary. We can see that total number of events is equally assigned for each colour (except for the blue colour, which has a slightly lower representation compared to the others). Censored means that the event has occurred (i.e. user clicked on con- 
tinue button). Results indicate that, for instance, in presence of the black colour 39.5\% of participants clicked on continue, while only $23.5 \%$ for yellow colour. Red, white, blue and green colours received very similar results.

\begin{tabular}{|l|c|c|c|c|}
\hline Colour Type & \multirow{2}{*}{ Total N } & \multirow{2}{*}{$\begin{array}{c}\mathrm{N} \text { of } \\
\text { Events }\end{array}$} & \multicolumn{2}{|c|}{ Censored } \\
\cline { 4 - 5 } & & 46 & 30 & $39.5 \%$ \\
\hline BLACK & 76 & 54 & 26 & $32.5 \%$ \\
\hline RED & 80 & 55 & 25 & $31.3 \%$ \\
\hline WHITE & 80 & 52 & 16 & $23.5 \%$ \\
\hline YELLOW & 68 & 40 & 19 & $32.2 \%$ \\
\hline BLUE & 59 & 50 & 22 & $30.6 \%$ \\
\hline GREEN & 72 & 297 & 138 & $31.7 \%$ \\
\hline Overall & 435 & 29 & \\
\hline
\end{tabular}

Table 2. Table 3. Case Processing Summary

Next, we proceed by examining the cumulative survival functions for different intervention groups (i.e. colour types). This is a plot of the cumulative survival proportions against time for each colour type. When the group survival curve appears "above" another colour group's survival curve on the graph (see Figure 2), it is considered to be demonstrating a beneficial/advantageous effect. However, in our context if a survival curve is above another one, it indicates that it has lower impact on user's behaviors'. For instance, on Figure 2, black colour is above the yellow colour, which indicates that black colour has lower impact on the user's compliance as its survival curve shows black warning message significantly prolongs the time taken until participants chose exit option. Overall, survival graph is a first indication of the colour effects that needs to be further analyzed to understand if it is statistically significant.

In order to understand the typical time until participants experience the event (choose exit action), we need to analyse the means and medians for survival time table. Table 4 presents the mean and median survival time for each of the colour types. As the mean has far less importance than the median as a measure of central tendency, we focus on interpreting the median values and their $95 \%$ confidence intervals.

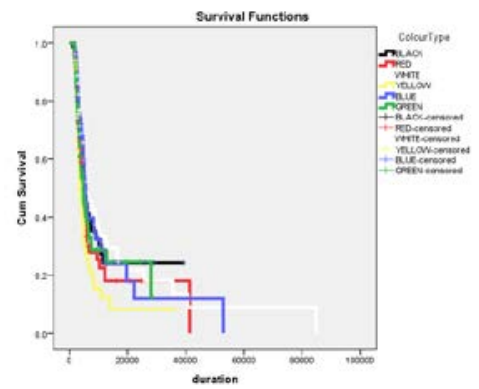

Fig. 2. Survival functions for each colour type 
We can see, for instance, that the median survival time for black colour was $6076 \mathrm{~ms}$ with $95 \%$ confidence interval from $4517 \mathrm{~ms}$ to $7634 \mathrm{~ms}$. The results from Table 4 indicate that yellow (median value of 3635), green (4707) and red (4902), were the most successful colours at keeping participants from continuing with their non-compliant action (click on continue). Other colours: black (6076), white (6065) and blue (5691) were less successful in keeping participants from being noncompliant.

\begin{tabular}{|c|c|c|c|c|}
\hline \multirow{2}{*}{$\begin{array}{c}\text { Tolour } \\
\text { Type }\end{array}$} & \multicolumn{2}{|c|}{ Median } \\
\cline { 2 - 5 } & Estimate & \multirow{2}{*}{ Std. Error } & \multicolumn{2}{|c|}{ 95\% Confidence Interval } \\
\cline { 3 - 5 } & & & Lower Bound & Upper Bound \\
\hline RED & 4902.000 & 278.550 & 4356.042 & 5447.958 \\
\hline BLACK & 6076.000 & 795.073 & 4517.658 & 7634.342 \\
\hline WHITE & 6065.000 & 1171.784 & 3768.303 & 8361.697 \\
\hline YELLOW & 3635.000 & 357.149 & 2934.987 & 4335.013 \\
\hline BLUE & 5691.000 & 572.842 & 4568.229 & 6813.771 \\
\hline GREEN & 4707.000 & 440.012 & 3844.576 & 5569.424 \\
\hline Overall & 4938.000 & 170.864 & 4603.107 & 5272.893 \\
\hline
\end{tabular}

Table 3. Means and Medians for Survival Time

Further, in order to understand if there were differences in the survival distribution for the different colour types, there are three statistical tests that can be used to test whether the survival functions are equal. These are the log rank test [36], Breslow test [37] and the Tarone-Ware test [38]. Usually all three tests provide very similar results. For instance, the log rank test is testing the null hypothesis that there is no difference in the overall survival distributions between the groups (i.e. colour types) in the population. Table 5 displays the results of the three tests we performed.

\begin{tabular}{|l|r|r|r|}
\hline & Chi-Square & df & \multicolumn{1}{c|}{ Sig. } \\
\hline Log Rank (Mantel-Cox) & 12.015 & 5 & .035 \\
\hline Breslow (Generalized Wilcoxon) & 12.257 & 5 & .031 \\
\hline Tarone-Ware & 12.948 & 5 & .024 \\
\hline
\end{tabular}

Table 4. Test of equality of survival distributions for the different levels of Colour type.

As $\mathrm{p}<0.05$ for all three tests, we conclude that the survival distribution for the five colour types were statistically significantly different, $\chi 2(5)=12.015, \mathrm{p}<.05$ for the log rank test. This indicates that the survival functions for different colour applications are statistically different. 


\section{Discussion}

Colour seems to play an important role in this communication process occurring between the communication medium (i.e. the warning message) and the receiver (i.e. the user). We found that colour has an important role in the Cognitive-affective model of communication as it helps to establish a better relationship between the user and the communication medium. This supports the balance between relationship and action, between cognition and affect, and between message and medium [28]. In other words, our study results confirm that users may have different expectations when it comes to the way the message is presented to them. Some colours, such as blue, will have less effect on user's attention, while others (e.g. red) will provoke an increased arousal. This is in line with past studies which found blue to have "calming" effect [27] and red, projected onto a diffusing screen, to produce increased users' arousal [30]. Further, we provide evidence that arousal effect can be high or low, or that users can even switch from high to low arousal effect during different event occurrences. This is supported by the theory of psychological reversals [27]. Our finding suggests that users' will have higher or lower levels of the arousal effect, which will depend on how they feel about the colour that is displayed. While this finding was also supported by others [e.g. 11, 39], this is the first investigation that validated different colour applications in the warning context. This confirms a strong link between arousal and colour preference. Indeed, it was found that warm colours (e.g. red) will create more arousal and attention than cool colours (e.g. blue) [40]. Also, cool colours will elicit greater relaxation and pleasure than warm colours [41]. This effect seems to be valid also in the warning context arousal is linked to the colour application. Moreover, the importance of the communication medium is even more pronounced as cognition and affect will have high or low arousal effect on user's behaviour.

Our study has some practical implications. First, we found that different colour applications (black, blue, yellow, red, green, white) have different psychological arousal effects on the user. As expected, we found red to have a high arousal effect. However, we also found that yellow and green are as powerful as red to prevent users from committing a potentially harmful action. Alternately, black, white and blue were less successful to keep participants from being non-compliant. This finding provides important insight for practice as it gives clear evidence that other colours, such as yellow and green, can have high arousal effect on user's attention. It could be that these colours increase comprehension. Past studies found that when contrasting colours in health warnings on tobacco products, such as black letters on a white background, leads to easier reading process, which, consequently increases comprehension [42, 43]. This suggests that warning designers could think of using other colour applications when displaying warning message to the user.

We believe that future research should further seek to understand colour effects on user's compliance by incorporating additional event occurrences. Indeed, some colours could have a greater secondary effect during successive event occurrences. For instance, in the fifth occurrence red could be simply ignored. The fact that we considered only two cultures is a limitation of our study. Hence, future studies could extend this cultural exploration by adding other cultures to further understand the colour 
impact in various cultural settings. Another limitation of our study is that, as we used experimental setting and deception approach, we could not collect some background information about our participants, which could be very useful to better understanding of different study's findings.

\section{$7 \quad$ Conclusion}

This research aimed at understanding how colour affects users' decision-making processes in the context of warning banner messages. We found that colour plays an important role in the user's decision-making process when confronted with a warning message. Different user arousal effects are observed not only in different cultural settings, but also depending on the event occurrence. Overall, we advance understanding of the psychological process that precedes a decision - with a focus on the importance of colour.

\section{References}

1. Silic, M., Back, A.: Information Security and Open Source Dual Use Security Software: Trust Paradox. Open Source Software: Quality Verification, pp. 194-206. Springer (2013)

2. Silic, M., Back, A.: Identification and Importance of the Technological Risks of Open Source Software in the Enterprise Adoption Context. In: 12th International Conference on Wirtschaftsinformatik, pp. 1163-1176. Thomas O.; Teuteberg, F. (Hrsg.), (2015)

3. Silic, M., Back, A.: The Influence of Risk Factors in Decision-Making Process for Open Source Software Adoption. International Journal of Information Technology \& Decision Making 1-35 (2015)

4. Silic, M., Back, A., Silic, D.: Taxonomy of technological risks of open source software in the enterprise adoption context. Information and Computer Security 23, 570-583 (2015)

5. Silic, M.: Dual-use open source security software in organizations - Dilemma: Help or hinder? Computers \& Security 39, Part B, 386-395 (2013)

6. Elliot, A.J., Maier, M.A.: Color and psychological functioning. Current Directions in Psychological Science 16, 250-254 (2007)

7. Kaya, N., Epps, H.H.: Relationship between Color and Emotion: A Study of College Students. College Student Journal 38, 396 (2004)

8. Valdez, P., Mehrabian, A.: Effects of color on emotions. Journal of Experimental Psychology: General 123, 394 (1994)

9. Silic, M.: Understanding Colour Impact on Warning Messages: Evidence from US and India. In: CHI'14 Extended Abstracts on Human Factors in Computing Systems. ACM, (2016)

10. Elliot, A.J., Maier, M.A., Moller, A.C., Friedman, R., Meinhardt, J.: Color and psychological functioning: the effect of red on performance attainment. Journal of Experimental Psychology: General 136, 154 (2007) 
11. Cyr, D., Head, M., Larios, H.: Colour appeal in website design within and across cultures: A multi-method evaluation. International Journal of Human-Computer Studies 68, 1-21 (2010)

12. Cyr, D., Head, M.: Website design in an international context: The role of gender in masculine versus feminine oriented countries. Computers in Human Behavior 29, 1358-1367 (2013)

13. Cyr, D., Trevor-Smith, H.: Localization of Web design: An empirical comparison of German, Japanese, and United States Web site characteristics. Journal of the American society for information science and technology 55, 1199-1208 (2004) 14. Menon, S., Kahn, B.: Cross-category effects of induced arousal and pleasure on the Internet shopping experience. Journal of retailing 78, 31-40 (2002)

15. Bhatnagar, A., Misra, S., Rao, H.R.: On risk, convenience, and Internet shopping behavior. Communications of the ACM 43, 98-105 (2000)

16. Wogalter, M.S.: Purposes and scope of warnings. Handbook of Warnings. Lawrence Erlbaum Associates, Mahwah, NJ 3-9 (2006)

17. Silic, M., Barlow, J., Ormond, D.: Warning! A Comprehensive Model of the Effects of Digital Information Security Warning Messages. The 2015 Dewald Roode Workshop on Information Systems Security Research, IFIP, pp. 1-32. IFIP, Dewald (2015)

18. Coleman, S.: The Minnesota income tax compliance experiment: replication of the social norms experiment. Available at SSRN 1393292 (2007)

19. Goldstein, N.J., Cialdini, R.B., Griskevicius, V.: A room with a viewpoint: Using social norms to motivate environmental conservation in hotels. Journal of consumer Research 35, 472-482 (2008)

20. Schultz, P., Tabanico, J.J.: Criminal beware: a social norms perspective on posting public warning signs*. Criminology 47, 1201-1222 (2009)

21. Egilman, D., Bohme, S.: A brief history of warnings. Handbook of Warnings. Lawrence Erlbaum Associates, Mahwah, NJ 35-48 (2006)

22. Akhawe, D., Felt, A.P.: Alice in Warningland: A Large-Scale Field Study of Browser Security Warning Effectiveness. In: Usenix Security, pp. 257-272. (2013) 23. Sunshine, J., Egelman, S., Almuhimedi, H., Atri, N., Cranor, L.F.: Crying Wolf: An Empirical Study of SSL Warning Effectiveness. In: USENIX Security Symposium, pp. 399-416. (2009)

24. Nema.: http://www.nema.org/Standards/z535/Pages/default.aspx, Retreieved on January 2016

25. Laughery, K.R., Young, S.L., Vaubel, K.P., Brelsford Jr, J.W.: The noticeability of warnings on alcoholic beverage containers. Journal of Public Policy \& Marketing 38-56 (1993)

26. Egelman, S., Schechter, S.: The importance of being earnest [in security warnings]. Financial cryptography and data security, pp. 52-59. Springer (2013)

27. Walters, J., Apter, M.J., Svebak, S.: Color preference, arousal, and the theory of psychological reversals. Motivation and emotion 6, 193-215 (1982)

28. Te'eni, D.: Review: A cognitive-affective model of organizational communication for designing IT. MIS quarterly 25, 251-312 (2001) 
29. Levy, B.I.: Research into the psychological meaning of color. American Journal of Art Therapy (1984)

30. Gerard, R.M.: Color and emotional arousal. In: American Psychologist, pp. 340340. AMER PSYCHOLOGICAL ASSOC 750 FIRST ST NE, WASHINGTON, DC 20002-4242, (1958)

31. Goldberg, J.H., Stimson, M.J., Lewenstein, M., Scott, N., Wichansky, A.M.: Eye tracking in web search tasks: design implications. In: Proceedings of the 2002 symposium on Eye tracking research \& applications, pp. 51-58. ACM, (2002) 32. Chiu, S.W., Gervan, S., Fairbrother, C., Johnson, L.L., Owen-Anderson, A.F., Bradley, S.J., Zucker, K.J.: Sex-dimorphic color preference in children with gender identity disorder: A comparison to clinical and community controls. Sex Roles 55, 385-395 (2006)

33. Russo, P., Boor, S.: How fluent is your interface?: designing for international users. In: Proceedings of the INTERACT'93 and CHI'93 conference on human factors in computing systems, pp. 342-347. ACM, (1993)

34. Kaplan, E.L., Meier, P.: Nonparametric estimation from incomplete observations. Journal of the American statistical association 53, 457-481 (1958)

35. Box-Steffensmeier, J.M., Jones, B.S.: Event history modeling: A guide for social scientists. Cambridge University Press (2004)

36. Mantel, N.: Evaluation of survival data and two new rank order statistics arising in its consideration. Cancer chemotherapy reports. Part 1 50, 163-170 (1966)

37. Breslow, N.: A generalized Kruskal-Wallis test for comparing K samples subject to unequal patterns of censorship. Biometrika 57, 579-594 (1970)

38. Tarone, R.E., Ware, J.: On distribution-free tests for equality of survival distributions. Biometrika 64, 156-160 (1977)

39. Bonnardel, N., Piolat, A., Le Bigot, L.: The impact of colour on Website appeal and users' cognitive processes. Displays 32, 69-80 (2011)

40. Birren, F.: Color \& human response: aspects of light and color bearing on the reactions of living things and the welfare of human beings. Van Nostrand Reinhold Company (1978)

41. Guilford, J.P.: The affective value of color as a function of hue, tint, and chroma. Journal of experimental psychology 17, 342 (1934)

42. Nilsson, T.: Legibility of tobacco health messages with respect to distance. A report to the Tobacco Products Division of the Health Protection Branch of Health and Welfare Canada (1991)

43. Hammond, D.: Health warning messages on tobacco products: a review.

Tobacco control tc. 2010.037630 (2011) 